\title{
Abundances of vanadium and bromine in 3 Centauri $A^{\star}$ (Research Note)
}

\section{Additional odd-Z anomalies}

\author{
C. R. Cowley ${ }^{1}$ and G. M. Wahlgren ${ }^{2}$
}

\author{
1 Department of Astronomy, University of Michigan, Ann Arbor, MI 48109-1042, USA \\ e-mail: cowley@umich.edu \\ 2 Lund Observatory, Lund University, Box 43, 22100 Lund, Sweden \\ e-mail: glenn.wahlgren@astro.lu.se
}

Received 5 August 2005 / Accepted 19 September 2005

\section{ABSTRACT}

We report abundance excesses of 1.2 and 2.6 dex, respectively, for vanadium and bromine in the hot, peculiar star 3 Cen A. Abundances for these two odd- $Z$ elements have not been previously reported for this star. Taken with previous work, they strengthen the case of the origin of the abundance peculiarities by diffusion.

Key words. stars: chemically peculiar - stars: abundances

\section{Introduction}

A dwindling number of the chemical elements lack robust identifications in stellar spectra. Moore (1945, cf. Table 6) omitted several elements from the Multiplet Tables as not being - for that time-of astrophysical interest. Among these elements was $Z=35$, bromine. Many of the elements listed in Moore's Table 6 have subsequently been identified in stars. Only recently, Castelli \& Hubrig (2004) identified bromine in the mercurymanganese (HgMn) star HR 7143 (HD 175640), based on three remarkably weak features. We note that Bidelman (2004) has suggested the presence of $\mathrm{Br}$ I in the ultra-peculiar spectrum of HD 101065, also known as Przybylski's star. In this work, we report the identification of lines of $\mathrm{Br}$ II in the spectrum of 3 Centauri A (HR 5210, HD 120709). The lines are significantly stronger than in HR 7143, though still rather weak, all less than $6.5 \mathrm{~mA}$.

We also report the unequivocal identification of V II and abundance determination of vanadium, a spectrum identified in many stars, but for which we have only found an upper limit previously reported for $3 \mathrm{Cen} \mathrm{A}$.

The findings of bromine and vanadium $(Z=23)$ are of significance from the point of view of the origin of abundance anomalies in hot, chemically peculiar (CP) stars of the upper main sequence. The basic mechanism postulated to account for these anomalies is a slow diffusive separation of the elements, depending on whether radiation pressure pushes them upwards,

\footnotetext{
* Based on ESO spectra from the UVESPOP project.
}

or they sink under the star's gravity. Typically, elements with high abundances - prior to diffusion - are less likely to be supported by radiation pressure than those with low abundances. This is because the lines of abundant elements are more likely to be saturated, so the radiation flux through them, and hence the radiation pressure on them would be less. This can give rise to an inverse odd-even effect in the theoretical predictions. Some odd- $Z$ elements, being typically of lower cosmic abundance than their even- $Z$ congeners, have larger predicted enhancements. The pattern can vary for elements in different parts of the periodic table.

Perhaps because of complicating factors, this inverse oddeven effect is not widely observed. However, some notorious cases can be found in the $\mathrm{HgMn}$ subgroup of the CP stars. In particular, the element gallium $(Z=31)$ shows huge enhancements, while its even- $Z$ neighbors zinc and germanium are weak or absent. For 3 Cen A, we can now add abundance enhancements of the odd- $Z$ elements vanadium and bromine.

The 3 Cen A spectrum (B5 III-IVp) has been rather well studied (Adelman \& Pintado 2000; Castelli et al. 1997; Wahlgren \& Hubrig 2004). The star is usually regarded as a member of the He-weak group of CP stars. It has abundance excesses of $\mathrm{P}(Z=15)$, Sc $(Z=21), \mathrm{Mn}(Z=25)$, and especially $\mathrm{Ga}(Z=31)$, anomalies that are shared with many $\mathrm{HgMn}$ stars. It is also one of the stars where the dominant helium isotope is ${ }^{3} \mathrm{He}$ (cf. Hartoog \& Cowley 1979).

Our study makes use of the high-quality spectra available from the ESO UVES Paranal Observatory Project archive 
(Bagnulo et al. 2004, henceforth UVESPOP). Spectra were downloaded from the web site. The resolution is "about 80000 ". We estimate $S / N$ for the unfiltered ("individual reduced") spectra to be between 100 and 150 .

Two approaches to data analysis were undertaken for comparison, both employing the Kurucz (1993) ATLAS9/WIDTH9/SYNTHE suite of programs with updated (3 Cen A) abundances.

In one approach the merged UVESPOP ascii wavelengthflux data were normalized, mildly Fourier filtered, and 2852 stellar features were measured (by CRC using programs developed at the University of Michigan). The software records both wavelengths and the central intensities of the features. The measurements were not made automatically; each required a human judgment; the reality of the faintest measurements is often moot. Stellar wavelengths ranged from 3070 to $10390 \AA$. A subset of the measurements, excluding lines longer than $1 \mathrm{mi}-$ cron were then subjected to analysis by wavelength coincidence statistics (WCS, cf. Cowley \& Hensberge 1981). The spectra of Br II and V II were found in this survey. Equivalent widths were measured by fitting individual Voigt profiles to the stellar absorption features, and abundances obtained from WIDTH9.

The second approach relied on synthetic spectrum modeling using two UVESPOP "individual reduced spectra". The star is not known to be variable, and we found no indication of variability in the spectra discussed here.

The spectrum synthesis was conducted (in Lund by GMW) using the SYNTHE program and a model atmosphere generated using the ATLAS9 code. The model and starting set of elemental abundances is that used by Wahlgren \& Hubrig (2004). Included into the synthetic spectrum analysis were the hyperfine structures for lines of both elements.

Both analyses adopted a model with $T_{\text {eff }}=17500 \mathrm{~K}$ and $\log (g)=3.8$ as well as the overall abundances from the sources cited.

\section{Bromine}

Table 1 shows laboratory and stellar data for Br II. The laboratory data come from Sansonetti, Martin, \& Young's (2005) list of strong lines. Intensities marked with a "P" are called persistent lines. Stellar wavelengths $\left(\lambda^{*}\right)$ are in Col. 4 , given here to the fraction of an angstrom. A dash indicates that no wavelength was measured near a laboratory wavelength. Weak features may or may not be measured, depending on rather complex subjective factors. Classified lines, or lines whose levels are known, are indicated by a "y" in Col. 2.

A WCS test with a tolerance window of $0.07 \AA$, the largest tolerance in the table, shows that the significance level of seven coincidences out of the 14 wavelengths sought is 0.00002 on the basis of 50000 Monte Carlo trials. This means that in just one of the 50000 trials were seven or more coincidences within $\pm 0.07 \AA$ found on a set of 14 nonsense wavelengths randomly placed near the laboratory positions. We consider the bromine identification to be beyond doubt from this statistic.

Stellar absorptions near all the laboratory positions were examined and equivalent widths measured or, for the weakest
Table 1. Br II lines studied.

\begin{tabular}{ccccccc}
\hline \hline Int. & Cla & $\begin{array}{c}\lambda \\
\AA\end{array}$ & $\begin{array}{c}\lambda^{*} \\
\AA\end{array}$ & $\begin{array}{c}W_{\lambda} \\
\mathrm{m} \AA\end{array}$ & $\log g f$ & $\begin{array}{c}\text { Abund } \\
\log (\mathrm{Ab} / \mathrm{Sum})\end{array}$ \\
\hline 500 & $\mathrm{n}$ & 3914.38 & - & 2.3 & & \\
500 & $\mathrm{n}$ & 3980.38 & .33 & 3.9 & & \\
1000 & $\mathrm{n}$ & 4233.89 & - & $<1.0$ & & \\
1000 & $\mathrm{n}$ & 4365.63 & .64 & 6.1 & & \\
500 & $\mathrm{n}$ & 4542.89 & - & $<1$ & & \\
500 & $\mathrm{n}$ & 4678.70 & - & $<1$ & & \\
$500 \mathrm{P}$ & $\mathrm{y}$ & 4704.86 & .85 & 6.3 & +0.408 & -6.79 \\
$500 \mathrm{P}$ & $\mathrm{y}$ & 4785.49 & .47 & 4.7 & +0.208 & -6.72 \\
$500 \mathrm{P}$ & $\mathrm{y}$ & 4816.67 & .71 & 2.3 & +0.060 & -6.91 \\
400 & $\mathrm{y}$ & 4930.62 & - & 1.3 & & \\
500 & $\mathrm{n}$ & 5182.36 & .36 & 4.4 & & \\
500 & $\mathrm{y}$ & 5238.26 & .30 & $<1$ & & \\
500 & $\mathrm{y}$ & 5332.07 & - & 1.9 & & \\
500 & $\mathrm{n}$ & 5506.72 & - & $<1$ & & \\
\hline
\end{tabular}

features, estimated. The most questionable feature is an unclassified line, $\lambda 4542.89$, which is missing, or just short of a local maximum.

Natural bromine consists of two isotopes found in the percentages of ${ }^{79} \mathrm{Br}:{ }^{81} \mathrm{Br}=50.69: 49.31$ (Rosman \& Taylor 1998). As both isotopes are of odd atomic number we must be careful when fitting line profiles to account for hyperfine structure (hfs). This requires both the determination of wavelengths for individual components of the structure and the distribution of the total oscillator strength over these components. Unfortunately, relatively little experimental work has been conducted on the spectrum of $\mathrm{Br}$ II. The two isotopes of bromine have the same nuclear spin $(I=3 / 2)$ and similar magnetic moments. An isotope shift for $\mathrm{Br} \mathrm{I} \lambda 6122$ has been measured to be about $2.5 \mathrm{~m} \AA$ (Tolansky 1932), but no IS has been observed in $\mathrm{Br}$ II lines (Ranade 1951). Therefore, IS is ignored in this analysis.

Abundances are based on three lines for which transition probabilities are available (Bengston \& Miller 1976). The lines are located at air wavelengths 4704.862, 4785.494, $4816.669 \AA$ and are transitions connecting the upper levels $4 \mathrm{p}^{3}\left({ }^{4} \mathrm{~S}^{\circ}\right) 5 \mathrm{p}{ }^{5} \mathrm{P}_{3,2,1}$, respectively, to the lower level $4 \mathrm{p}^{3}\left({ }^{4} \mathrm{~S}^{\circ}\right) 5 \mathrm{~s}$ ${ }^{5} \mathrm{~S}_{2}{ }^{\circ}$. Bengston and Miller estimate their errors to be $35 \%$.

Results from ATLAS9/WIDTH9 are given in the final column of Table 1 . They are based on the average of four equivalent width measurements, two at Lund and two at Michigan. A straight average of the logarithms is $-6.81 \pm 0.10$, using a microturbulence of $\xi_{t}=1.0 \mathrm{~km} \mathrm{~s}^{-1}$. The difference in results using $\xi_{t}=0$ and $\xi_{t}=2$ ranges from 0.01 to $0.02 \mathrm{dex}$ for the three lines. We consider this difference insignificant at the current level of accuracy.

In the spectral synthesis, hyperfine structure was incorporated for the wavelengths and oscillator strengths using the magnetic hyperfine $A$ constants measured by Ranade (1951). Spectra were generated using the SYNTHE program (Kurucz 1993). Figure 1 presents these results for the case of $v \cdot \sin (i)=$ $2.0 \mathrm{~km} \mathrm{~s}^{-1}$ and a bromine abundance of -6.8 , claimed to be the best fit for the $\mathrm{Br}$ II lines. For each of the two segments (437B_a, 437B_b) of the observation the same two synthetic 


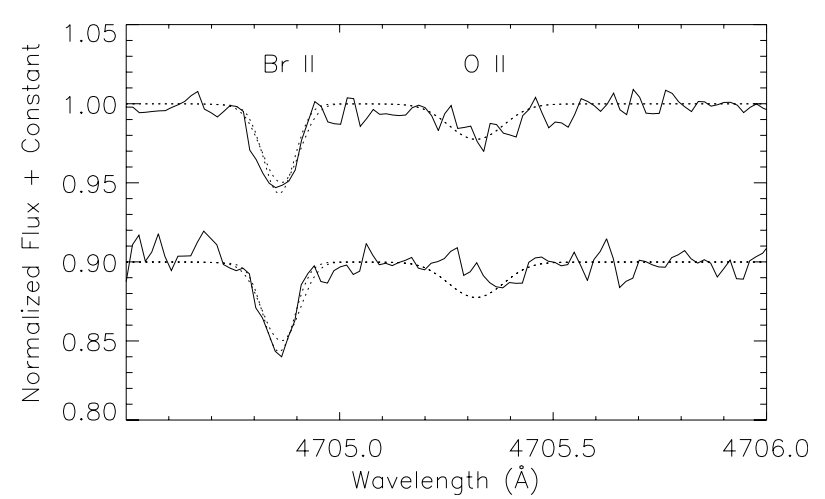

Fig. 1. Br II in the spectrum of 3 Cen A. The observation (solid) is compared with synthetic spectrum calculations (dashed). Upper and lower comparisons are for the two spectra of the observation. See text for details.

spectra are compared; accounting for the inclusion and exclusion of hfs. It can be seen that the hfs does broaden this $\mathrm{Br}$ II line at the expense of making the profile more shallow by an amount that is equivalent to about $0.1 \mathrm{dex}$. The seemingly good fit to the lower observation in the panel by the hfs excluded line profile is, of course, illusory, and it can be seen that the two spectra are noise affected.

The "solar" value, based on CI chondrites, is -9.4 (Lodders 2003; Asplund et al. 2004). The bromine excess in 3 Cen A from both methods is thus 2.6 dex, only slightly less than the excesses found by Castelli et al. for Ga and $\mathrm{Kr}$. This abundance enhancement is computed under the assumption that both isotopes are present in the stellar atmosphere.

Elemental abundance and isotopic anomalies are common for chemically peculiar stars of the upper main sequence. For 3 Cen A, isotope anomalies are inferred from line profiles for the elements He (Sargent \& Jugaku 1961) and Hg (Wahlgren $\&$ Hubrig 2004), and may well exist for other elements. In the event that the isotopic composition of $\mathrm{Br}$ in the atmosphere is that of only one isotope, the abundance enhancement - for that isotope - would be roughly doubled.

\section{Vanadium}

The strongest V II lines lie short of the traditional photographic region (roughly $\lambda \lambda 3750-4650$ ). Table 2 shows our results for $31 \mathrm{~V}$ II lines. The lines are primarily strong transitions from Meggers et al. (1975). Wavelengths and transition probabilities are from the VALD database (Ryabchikova et al. 1999). Details for individual lines may be found at the VALD website. The primary reference for oscillator strengths is Biemont et al. (1989).

Many of the equivalent widths were measured twice at Lund and twice at Michigan. The number of measurements is shown in the column headed "No". Those lines with fewer than four measurements reflect a variety of circumstances related to blending and the quality of the data used at Michigan and at Lund. Stellar wavelengths are given in the column headed $\lambda^{*}$, with only the fraction of an angstrom shown. We consider $\pm 0.02 \AA$ to be an acceptable deviation of the stellar and laboratory position. Larger deviations are flagged with an asterisk;
Table 2. V II lines studied.

\begin{tabular}{|c|c|c|c|c|c|c|}
\hline \multirow{2}{*}{$\begin{array}{l}\lambda \\
\AA\end{array}$} & \multirow{2}{*}{$\begin{array}{c}\lambda^{*} \\
\AA \\
\AA\end{array}$} & \multirow{2}{*}{$\begin{array}{r}W_{\lambda} \\
\mathrm{m} \AA \\
\end{array}$} & \multirow[t]{2}{*}{ No. } & \multirow[t]{2}{*}{$\log g f$} & \multicolumn{2}{|c|}{$\log (\mathrm{Ab} / \mathrm{Sum})$} \\
\hline & & & & & $\xi_{t}=0$ & $\xi_{t}=2$ \\
\hline 3093.105 & 0.09 & 21.2 & 4 & 0.599 & -6.57 & -6.72 \\
\hline 3094.216 & $0.20^{a}$ & 2.8 & 4 & -0.768 & -6.93 & -6.95 \\
\hline 3102.289 & 0.30 & 14.4 & 4 & 0.448 & -6.77 & -6.85 \\
\hline 3110.700 & 0.71 & 7.6 & 4 & 0.340 & -7.07 & -7.10 \\
\hline 3118.373 & 0.39 & 7.6 & 4 & 0.196 & -6.93 & -6.97 \\
\hline 3121.136 & 0.16 & 2.9 & 3 & -0.429 & -6.78 & -6.79 \\
\hline 3122.908 & 0.90 & 5.2 & 2 & 0.160 & -6.16 & -6.18 \\
\hline 3125.276 & $0.27^{b}$ & 13.6 & 4 & 0.044 & -6.42 & -6.49 \\
\hline 3126.211 & 0.19 & 5.6 & 4 & -0.268 & -6.62 & -6.64 \\
\hline 3130.261 & 0.25 & 3.8 & 3 & -0.285 & -6.81 & -6.82 \\
\hline 3133.327 & 0.31 & 3.5 & 3 & -0.484 & -6.65 & -6.67 \\
\hline 3134.933 & 0.94 & 3.7 & 4 & 0.050 & -6.35 & -6.37 \\
\hline 3188.514 & 0.51 & 4.8 & 4 & 0.089 & -6.78 & -6.80 \\
\hline 3190.683 & 0.70 & 11.4 & 2 & 0.262 & -6.45 & -6.51 \\
\hline 3217.113 & 0.09 & 4.1 & 4 & 0.198 & -6.69 & -6.71 \\
\hline 3254.762 & 0.77 & 2.6 & 3 & -0.042 & -6.68 & -6.69 \\
\hline 3267.704 & 0.70 & 6.1 & 4 & 0.283 & -6.84 & -6.87 \\
\hline 3271.123 & $0.11^{c}$ & 6.5 & 2 & 0.377 & -6.89 & -6.92 \\
\hline 3517.296 & $0.34 * d$ & 4.5 & 2 & -0.208 & -6.44 & -6.46 \\
\hline 3545.194 & 0.19 & 1.9 & 2 & -0.259 & -6.82 & -6.82 \\
\hline 3556.792 & 0.81 & 2.4 & 4 & -0.066 & -6.89 & -6.90 \\
\hline 3589.749 & $0.70 *$ & 1.8 & 2 & -0.295 & -6.81 & -6.81 \\
\hline 3715.466 & $0.44^{e}$ & $1.9-$ & 2 & -0.250 & -7.20 & -7.20 \\
\hline 3727.343 & $0.33^{c}$ & 2.5 & 3 & -0.231 & -7.05 & -7.06 \\
\hline 3815.388 & 0.36 & 1.9 & 2 & -0.400 & -6.58 & -6.58 \\
\hline 3847.339 & $0.38 *$ & $1.5-$ & 4 & -0.608 & -6.53 & -6.53 \\
\hline 3878.704 & 0.72 & $3.5-$ & 3 & -0.609 & -6.47 & -6.48 \\
\hline 3899.129 & 0.12 & $2.5-$ & 2 & -0.784 & -6.46 & -6.46 \\
\hline 3903.262 & 0.29 & $1.9-$ & 2 & -0.938 & -6.54 & -6.55 \\
\hline 4005.705 & 0.70 & 2.9 & 4 & -0.522 & -6.64 & -6.65 \\
\hline 4023.378 & 0.40 & 2.1- & 2 & -0.689 & -6.63 & -6.63 \\
\hline
\end{tabular}

Notes: ${ }^{a}$ Asymmetric; ${ }^{b}$ Broad; ${ }^{c}$ Poor continuum; ${ }^{d}$ blend; ${ }^{e}$ Balmer wing; * wavelength discrepancy; - equivalent width augmented (see text).

they usually indicate blending, or a setting error. All equivalent widths were measured with respect to the local continuum; those marked with a minus sign have been augmented slightly to compensate for lowering of the local continuum by blends. For example, the largest such change was for $\lambda 3847$, where the equivalent widths was increased by $7 \%$ because the continuum was depressed by $7 \%$ by the wing of $\mathrm{H} 9$.

Abundances based on the equivalent width measurements are presented for two values of the microturbulent velocity $\left(\xi_{t}\right)$. Averages of the $\log$ arithms yield $\log (V / \mathrm{Sum})=-6.7 \pm 0.2$ for the two microturbulences. A subset the 5 lines from the principal diagonal of V II Multiplet 1 yields an abundance of -6.8, about 0.1 dex lower. These are the lines selected for spectral synthesis at Lund.

Terrestrial vanadium has two stable isotopes in the proportions ${ }^{50} \mathrm{~V}:{ }^{51} \mathrm{~V}=0.250: 99.750$ (Rosman \& Taylor 1998). The even-A isotope has been ignored in our calculations. The oddA isotope possesses hfs for its line profiles. The hfs $A$ constants in V II have been determined by Arvidsson (2003) for 26 levels 


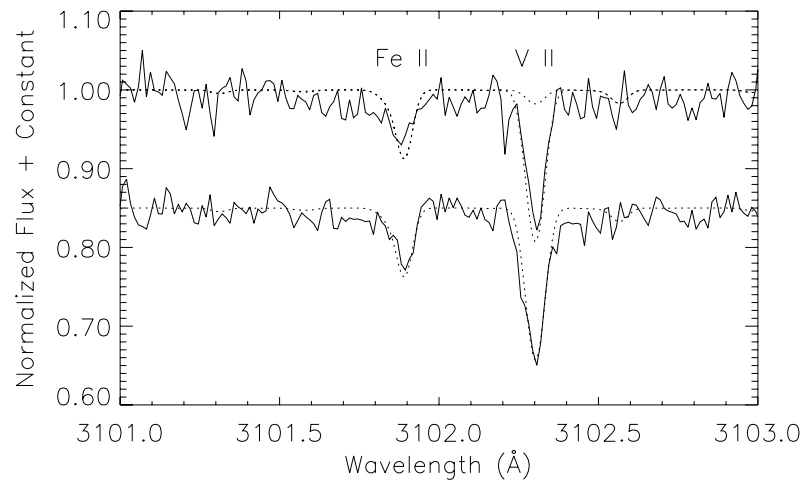

Fig. 2. V II in the spectrum of 3 Cen A. The observation (solid) is compared with the synthetic spectrum calculation for best fit abundance (upper and lower) and the solar abundance of vanadium (upper only).

from laboratory Fourier transform spectroscopy experiments, with hfs components and their relative intensities tabulated.

Five lines of V II in our stellar spectrum $(\lambda \lambda 3093.105$, $3102.289,3110.700,3118.373,3125.276$ ) have available hfs data, and could be included into our synthetic spectrum calculations. All five lines are well fit with an abundance of $-6.84 \pm 0.05$.

The scatter from the equivalent widths is much larger than from the synthesis, mostly resulting from noise and line blending. The hfs does not make a noticeable effect upon these particular lines.

Figure 2 presents a comparison of the observed data with synthetic spectra for the line V II $\lambda 3102$ for the two spectra of observation segment 346B.The upper comparison in the figure also includes the synthetic spectrum computed with the solar abundance of vanadium.

We adopt a vanadium abundance of -6.84 , giving a slight preference to the profile fitting of the stronger lines of Multiplet 1. This is an excess of 1.2 dex above the corresponding solar value (-8.0). It is similar to the excesses reported by Adelman \& Pintado (2000) or Castelli et al. (1997) for the odd$Z$ elements $\mathrm{P}, \mathrm{Sc}$, and $\mathrm{Mn}$ in $3 \mathrm{Cen} \mathrm{A}$.

\section{Conclusions}

The numerous odd- $Z$ anomalies of 3 Cen $A$, along with the dominance of ${ }^{3} \mathrm{He}$, make this object a paradigm example of chemical separation in stars. A comprehensive study that would simultaneously account for all of these peculiarities would have great value.

Acknowledgements. C.R.C. gratefully acknowledges help from Dr. R. L. Kurucz with the WIDTH code. Thanks are also due to the ESO staff for the UVESPOP public data archive.

\section{References}

Adelman, S. J., \& Pintado, O. I. 2000, A\&A, 354, 899

Arvidsson, K. 2003, Master Thesis, Lund Observatory, Lund University

Asplund, M., Grevesse, N., \& Sauval, A. J. 2004, in Cosmical Abundances as Records of Stellar Evolution and Nucleosynthesis, ed. F. N. Bash \& T. G. Barns, ASP Conf. Ser., in press, [arXiv:astro-ph/0410214] v2 10 Oct. 2004

Bagnulo, S., Jehin, E., Ledoux, C., et al. 2004, http://www.sc.eso.org/santiago/uvespop/

Bengston, R. D., \& Miller, M. H. 1976, Phys. Rev. A, 14, 1915

Bidelman, W. P. 2004, in Cosmical Abundances as Records of Stellar Evolution and Nucleosynthesis, ed. F. N. Bash, \& T. G. Barns, ASP Conf. Ser., in press

Biemont, E., Grevesse, N., Faires, L. M., et al. 1989, A\&A, 209, 391

Hartoog, M. R., \& Cowley, A. P. 1979, ApJ, 228, 229

Castelli, F., \& Hubrig, S. 2004, A\&A 425, 263

Castelli, F., Parthasarathy, M., \& Hack, M. 1997, A\&A, 321, 254

Cowley, C. R., \& Hensberge, H. 1981, ApJ, 224, 252

Kurucz, R. L. 1993, Atlas9 Stellar Atmospheres Programs and 2 km/s grid Krucuz, CD-Rom, No. 13, Cambridge MA: Smithson- ian Ap. Obs.

Lodders, K. 2003, ApJ, 591, 1220

Meggers, W. F., Corliss, C. H., \& Scribner, B. F. 1975, Tables of Spectral Line Intensities, Part I, NBS Monograph 145 (US Gov. Print. Office: Washington)

Moore, C. E. 1945, Cont. Princeton Univ. Obs., No. 20

Ranade, J. D. 1951, Phil. Mag., 326, 284

Rosman, \& Taylor, P. D. P. 1998, Pure. Appl. Chem., 70, 217 (IUPAC Technical Report)

Ryabchikova, T. A., Piskunov, N. E., Stempels, H. C., Kupka, F., \& Weiss, W. W. 1999, Proc. of the 6th International Colloquium on Atomic Spectra and Oscillator Strengths, Victoria BC, Canada, 1998, Phys. Scr. T, 83, 162

Sansonetti, J. E., Martin, W. F., \& Young, S. L. 2005, Handbook of Basic Atomic Spectroscopic Data (version 1.1.1), http://physics.nist.gov/PhysRefData/Handbook/ index.html

Sargent W. L. W., \& Jugaku, J. 1961, ApJ, 157, 757

Tolansky, S. 1932, Proc. Royal Soc. A, 136, 585

Wahlgren, G. M., \& Hubrig, S. 2004, A\&A, 418, 1073 